\title{
Non-Viral Vehicles: Principles, Applications, and Challenges in Gene Delivery
}

\author{
Abbas Padeganeh ${ }^{1}$, Mohammad Khalaj-Kondori², \\ Babak Bakhshinejad ${ }^{1}$ and Majid Sadeghizadeh ${ }^{1}$ \\ ${ }^{1}$ Department of Genetics, Faculty of Biological Sciences, \\ Tarbiat Modares University, Tehran \\ ${ }^{2}$ Department of Biology, Faculty of Natural Sciences, University of Tabriz, Tabriz,
}

Iran

\section{Introduction}

Gene therapy is often referred to as transfer of transgenes into the somatic cells of a patient to obtain a therapeutic effect .One of the goals for all such therapies is efficient and safe delivery of the desired extrinsic genes into target cells, thereby increasing the therapeutic efficiency (Robson \& Hirst, 2003). This has been a major obstacle in gene therapy experiments (Sarbolouki et al., 2000; Sadeghizadeh et al., 2008).

To address this problem, there has been an increasing number of reports in the development of efficient gene delivery vehicles in recent years (Sadeghizadeh et al., 2008). Clinical trials have also focused on the delivery of genes directly to the target area e.g. tumor sites by intratumoral administration of both viral and non-viral delivery agents. But the problem remains to be overcome yet, as majority of tumors are not accessible for direct injection. A number of strategies are now being developed to target viral and non-viral delivery agents to tumor sites. These include genetically modifying viral carriers and incorporating a novel tumor-specific ligand into the viral coat proteins to direct the viral system to a tissue receptor and also incorporation of tissue specific ligands and monoclonal antibodies onto the surface of non-viral carriers (Robson \& Hirst, 2003). The delivery of the carrier system to the target site is however not the end of the goal. Efficient entrance of the gene or drug into the cells and expression of therapeutic gene are also the next hurdles to be overcome. There are several techniques for delivery of genes as well as drugs into eukaryotic cells using similar carriers practiced in-vitro and in-vivo. The in-vivo efficacy of a gene or drug delivery system depends on its capability to pass the main extracellular and intracellular barriers encountered from the site of administration to entry into the nucleus of desired cells (Sarbolouki et al., 2000; Sadeghizadeh et al., 2008).

The therapeutic effect of a gene therapy experiment would be expected once the introduced transgene in target cells is considered as part of the genetic component of host cell and leads to the production of a new functional protein. To date, this type of gene transfer known as transfection (Lewin, 2007; Singleton \& Sainsbury, 1995) has been studied widely and various techniques have been developed for it, each possessing its own advantages and shortcomings. Generally speaking, gene delivery techniques are classified into viral and 
non-viral categories. Non-viral systems include physical, chemical and biological methods. Conventional physical techniques include electroporation and microinjection. Among chemical methods are calcium-phosphate precipitation, use of diethylaminoethyl (DEAE), polyethylenimines (PEIs), polybrene/dimethyl sulfoxide, liposomes, cationic amino polymers, polyamidoamin (PAMAM), dendrimers and dendrosomes (Guyden, 1993; Hammarskjold, 1991). Biological methods embrace viral transfer systems which utilize SV40-based vectors, adenoviral vectors, vaccina virus vectors, BPV vectors and retroviral carriers (Hammarskjold, 1991; Wong \& Neuman, 1982) and non-viral systems which bacteriophages are among the most important. A detailed description all of these delivery systems lies beyond the scope of this paper (for review see references Hammarskjold, 1991; Wong \& Neuman, 1982).

Nanotechnology referred to as the creation of useful materials, devices and systems used to manipulate matter at an extremely small size -between 1 and $100 \mathrm{~nm}$ - (Alivisatos, 1996; Suntherland, 2002) offers great opportunity in the field of drug and gene delivery. The problems and shortcomings of current anti-cancer treatment strategies such as systemic administration of drugs or genes which do not greatly differentiate between cancerous and normal cells leading to systemic toxicity and adverse effects, have caused limitations in allowable dose of drugs (Sinha et al., 2006) and led to a resurgence of interest in developing safe and efficient nano-scale gene and drug porters capable of detecting target sites and delivering proper genes and/or drugs to diseased cells (Sadeghizadeh et al., 2008).

In recent years, a number of nanoparticle-based therapeutic agents have been developed for treatment of cancer, diabetes, asthma, allergy, infection, etc. (Brannon-Peppas \& Balanchette, 2004; Kawasaki \& Player, 2005) . These nano-scale agents may provide more efficient and/or more convenient routes of administration, have lower toxicity, extend the product life-cycle and ultimately allow targeted and controlled release of therapeutic gene or drug (Zhang et al., 2007).

It has previously been reported that dendrosomes are capable of easily delivering genes into human cells (e.g. human hepatocytes, kidney cells and several cancer cell lines) and animal models in-vivo. They are easily synthesized, highly stable (nearly 4 years at ambient conditions) and extremely convenient to handle and use (Sarbolouki et al., 2000; Sadeghizadeh et al., 2008).

In this chapter, we discuss some of the most commonly used non-viral gene delivery systems (some also used as drug-carriers), with a focus on features of the recently introduced dendrosomes as novel gene porters shedding more light onto future perspectives of these promising nanocarriers.

Non-viral biological gene delivery methods include bacteria, bacteriophages, virus-like particles (VLPs), erythrocyte ghosts and exosomes. Elaboration on all of these approaches is beyond the scope of this chapter. Unavoidably, in this regard we will narrow our debate to bacteriophages being one of our research interests. Hence, at the end of the chapter, bacteriophages as one of the most significant non-viral biological systems or strategies for gene delivery developed over the recent years will be discussed (Seow \& Wood, 2009).

\section{Chemical strategies}

\subsection{Calcium-phosphate precipitation}

This technique is the most common way to transfect foreign genes into eukaryotic cells mainly due to its simplicity and inexpensiveness. In this technique, foreign genes are 
precipitated on the surface of cell monolayer. Briefly, calcium chloride, DNA and phosphate buffer are mixed at a neutral $\mathrm{pH}$, the calcium-phosphate-DNA complex then precipitates on the cells and would enter the cells through endocytosis. These complexes are then transported to different organelles including the nucleus (Guyden, 1993). In order to increase the efficiency of transfection, addition of glycol/dimethyl sulfoxide to the monolayer following precipitation of the complex and removal of the old medium has been effective (Okayama et al., 1991; Li \& Thacker, 1997). However, this method suffers from frailties including transient and unstable expression of tranfected genes following their degradation, low percentage of stably-transformed cells (only $0.001-1 \%$ ) and the need to determine the optimum conditions for transfectoin of each cell type.

Nevertheless, calcium phosphate nanoparticles introduced by Roy et al. (2003) are ultra-low size entities around $80 \mathrm{~nm}$ in diameter and it seems to be able to protect encapsulated DNA from environmental DNases with capability of surface modifications. This new class of nanoparticles have been used for gene delivery to liver.

\subsection{Diethylaminoethyl (DEAE)-dextran}

This method is based upon the negative charge of DNA and the positive charge of diethyl aminoethyl-dextran leading to the formation of a complex and adherence of the complex to the cell surface followed by endocytosis. This technique benefits from advantages such as simple and rapid preparation steps, low cost and reproducibility. However, cells show different sensitivities to the toxicity of this compound, therefore the proper ratio of diethylaminoethyl-dextran-DNA must be determined for each cell line (Holter et al., 1989). Moreover, it is preferred for transfection of adherent cells over cells in suspension (Gulick, 2003).

\subsection{Polycations}

Many libraries perform DNA trasfection experiments using artificial polycations such as polybren/dimethyl sulfoxide which have been shown to enhance retroviral infection in cell cultures by making an electrostatic bridge between the negatively charged viral particles and neutral components of the recipient cell membrane. It also binds DNA and attaches it to the cell surface. Finally, exposing the cells to dimethyl sulfoxide increases the speed of DNA uptake. It is a proper method when dealing with very limited amounts of DNA (ng DNA). The efficiency of this technique is sometimes $0.01-0.1 \%$ (Roy et al., 2003). Other polycationbased systems may also utilize poly-lysine compounds (Sarbolouki et al., 2000). Of the most widely used gene carriers of this category are polyethylenimines (PEIs). Linear or branched PEIs have been efficiently used for in vitro transfection of genes. However, in vivo application of PEIs, leads to non-specific interactions of the PEI/DNA complex with components of the host blood and results in failure of proper gene delivery. Thus, various surface modified derivatives of PEIs (polyethylene glycol-conjugated PEIs) have been emerging to overcome these issues (Kichler, 2004).

\subsection{Polymeric L-lysin vehicles}

Poly L-lysin, also referred to as PLL, has been shown to form complexes with DNA as a result of interaction of negatively charged DNA and positively charged amino groups of lysine (Tae et al., 2006). These polyelectrolyte complexes have also been shown to suffer from drawbacks e.g. high degree of cytotoxicity, rapid clearance and self aggregation (Liu et 
al., 2001). Similar to that of PEIs, surface conjugation of PLL with polyethylene glycol, has been shown to improve properties of this class of gene delivery vehicles. In addition, various strategies have been employed to target PLL polymers to specific cell types or tissues. These include conjugating sugar moieties e.g. lactose or galactose to target PPL/DNA complexes to hepatocytes (Nishikawa et al., 1998; Hashida et al., 1998). There have also been efforts to conjugate antibodies (e.g. leukemia-specific- antigen antibody, anti JL-1 antibody) to PLL complexes showing higher leukemia-specific cell uptake and specificity (Suh et al., 2001).

\subsection{Polysaccharides as gene carriers}

There are known examples of using polymers composed of sugar molecules, e.g. chitosan and cyclodextrin, for gene delivery purposes. Cyclodextrin is in fact a cyclic entity made up of oligomeric glucose units forming a hydrophobic internal cavity and a hydrophilic extremity. Another example is chitosan, a polysaccharide made up of repetitive units of Dglucosamine linked to $\mathrm{N}$-acetyl-D-glucosamine. Both above mentioned polysaccharidebased structures can interact with DNA to form stable complexes and have been reported to have comparable or higher transfection efficiencies in regard to PEI or PLL (Gonzalez et al., 1999).

\section{Liposome-based gene/drug delivery systems}

Liposomes are spherical lipid vesicles with bilayer membrane structure composed of natural or synthetic amphiphilic lipid molecules (Zhang \& Granick, 2006). Liposomes have been widely used as pharmaceutical carriers in the past decade because of their unique abilities in encapsulating both hydrophilic and hydrophobic agents with a high efficiency, protecting the encapsulated drugs from undesirable side effects of external conditions, being functionalized with specific ligands that can target specific cells, and being coated with inert biocompatible polymers (Roy et al., 2003; Moghimi \& Szebeni, 2003). Liposomes are also used as gene carriers. An efficient strategy to encapsulate DNA within liposome is the reverse phase evaporation method (REV) in which phospholipids are resolved in ether making up an organic phase and DNA is added to PBS making up an aqueous phase. Then the aqueous and the organic phases are emulsified in a sonicator followed by mixing the lipids with DNA which leads to the formation of lipid vesicles containing the DNA molecules inside (Guyden, 1993). Since liposomes are usually not fused to the cell surface but rather phagocytosized by cells, the carried nucleotides would be exposed to the lysosomic enzymes and therefore digested, reducing the efficiency of successful transfection/expression process. Other problems of liposomes include the possibility of formation of small-sized liposomes uncapable of encapsulating large macromolecules such as DNA and the multistep difficult processes of their production. They also have a low gene transfer efficiency and usually exhibit cytotoxicity in lymphoma cells (Buttgereit et al., 2000).

\subsection{Cationic liposomes}

Cationic liposomes such as lipofectins have also been developed to overcome some of mentioned shortcomings of liposomes. Lipofectins contain positively charged lipids like dioleoylphosphatidylethanolamine (DOPE) and N-(1-2,3-dioleyloxypropyl)-N,N,Ntrimethylammonium (DOTMA). DOTMA for example, is designed as stable cationic bilayer 
vesicles spontaneously interacting with polyanionic DNA and RNA molecules and therefore forming liposome/polynucleotide complexes. These complexes are taken in by the anionic surface of host cells with an efficiency of about 10-100 fold higher than that of negatively or neutrally charged liposomes. Large DNA molecules such as baculoviral DNA $(130 \mathrm{~kb})$ and genomes of RNA-viruses have also been introduced into cell-cultures using DOTMA confirmed by the formation of viral particles (Felgner, 1991; Strauss et al., 1994).

\subsection{Modified and targeted liposomes}

One drawback of the use of liposomes is the fast clearance of liposomes from blood by phagocytic cells of the reticuloendothelial system, resulting in unfavorable therapeutic index (Roy et al., 2003). One of the widely used strategies is to formulate long-circulating liposomes by coating the liposome surface with inert biodegradable polymers such as polyethylene glycol. The polymer layer provides a protective shell over the liposome surface and suppresses liposome recognition by opsonins and therefore subsequent clearance by the reticuloendothelial system (Dutta et al., 2006). Another strategy is to increase the accumulation of liposomes in the target site by attaching targeting ligands such as antibodies and small moiety molecules (e.g. folate and transferrin) to the liposome surface. Targeted liposomes have been developed for differential drug and gene delivery (Saunak et al., 2004).

\section{Nanopolymer-based carriers}

\subsection{Dendrimers}

Dendrimers are a class of artificial, highly branched and reactive three dimensional polymers, with all bonds originating from a central core. The term dendrimer comes from the Greek word "dendron" which means tree and the suffix "mer" from meros referring to smallest repeating units. In recent years, there has been much interest in dendrimers; since due to the large number of terminal functional groups (e.g. amino groups) on their surface, they are easily linkable to antibodies and reactive therapeutic agents making them proper for use in biomedical research (Bousif et al., 1995; Buttgereit et al., 2001; Massumi et al., 2005). Other attractive features such as nanoscale size, highly controllable molecular weight and possibility of encapsulating a guest molecule (e.g. a gene or drug) in their internal cavities (Tomalia, 2005) give dendrimers a distinctive advantage over other polymers for delivery of drugs and genes (Strauss, 1994). To use DNA therapeutically, it must pass some barriers in the body of host, including capillary endothelial cells, phagocytes, reticuloendothelial system and eventually the membrane of the target cell (Dutta et al., 2006). The nanoscopic size of dendrimers not only helps them evade the reticuloendothelial system, but also generates benefit for them in intracellular delivery [34]. Amino-dendrimers have been specifically attractive due to their defined structures and the large number of surface amino groups ((Sarbolouki et al., 2000; Sadeghizadeh et al., 2008; Bielinska et al., 1997). Ployamidoamin (PAMAMs) dendrimers are a member of this family of dendrimers known as water soluble constructs covered with a large number of amino groups on their surface due to which they are positively charged at physiologic $\mathrm{pH}$ and therefore thought to interact with DNA (Sadeghizadeh et al., 2008; Kukowska et al., 1996) . Another member of this family "poly (propyleneimine) dendrimers" (PPI) are also highly branched and globular with primary amino groups on the periphery (Saunak, 2004). As a result, these dendrimers readily form complexes with DNA and are capable of transfecting cell cultures with low 
toxicity and higher efficiency. Dendrimers have different generations and modification of the large number of surface functional groups by conjugation to guest molecules has led to the production of dendrimer conjugates (Buttgereit et al., 2001; Massumi et al., 2005). For example, in a recent study, labeled biotin-conjugated PAMAM dendrimers were constructed and used to target tumor cells. As biotin specific receptors are overexpressed on the surface of cancer cells, results have shown an increased carrier uptake by these cells (Pourasgari et al., 2007).

There are few studies on the antigenic properties of nanoparticles such as dendrimers. An early study on PAMAM dendrimers did not reveal overt antigenicity of generations 3, 5 and 7 amino-terminated dendrimers (Dutta et al., 2006). In a study on immunosuppressive properties of dendrimers, generations 3 and 5 PAMAM dendrimers conjugated to glucosamine strongly inhibited induction of inflammatory cytokines and chemokines in human macrophages and dendritic cells exposed to bacterial endotoxins. Also, dendrimers have been reported to possess hemolytic toxicity and cytotoxicity owing to their cationic nature. Moreover, interaction with oppositely charged macromolecules in plasma may result in premature release of their cargo (e.g. plasmid DNA or carried drug) within the blood (Buttgereit et al., 2001). In addition, degradation of the plasmid DNA by plasma DNases leads to poor gene expression in-vivo (Massumi et al., 2005; Pourasgari et al., 2007).

\subsection{Dendrosomes: New generation of nanoscale gene porters}

Dendrosomes, are a novel family of non-viral vehicles and gene porters that form hyperbranched spherical entities hence the term derndrosome is applied to them (Sarbolouki et al., 2000; Sadeghizadeh et al., 2008).

Dendrimers could be presumably considered as the primary ancestors of these novel gene delivery systems. Dendrosomes possess valuable advantages over other carriers which include ease of synthesis, stability (nearly 4 years at ambient conditions), nontoxicity, inexpensiveness, biodegradability, neutrality, spherical structure, capability of easily delivering genes and being extremely convenient to handle and use (Sarbolouki et al., 2000; Sadeghizadeh et al., 2008; Dobrovolskaia \& McNeil., 2007).

According to atomic force microscopy (AFM) observations, dendrosomes are expectedly nanoscopic particles $10-100 \mathrm{~nm}$ in size . A unique feature of dendrosomes is the ease with which they provide suitable inert gene porters for various DNA sizes and target cells. There have been several reports by our group and other researchers all showing that dendrosomes may serve as promising high efficient candidates for transfection and therapy (Sarbolouki et al., 2000; Sadeghizadeh et al., 2008). In early studies, three generations of dendrosomes named Den450, 700 and 123 were synthesized and used and their applicability and efficiency were assessed by studies on transfection of human cell cultures as well as vaccination of mice against hepatitis B. To assess their cytotoxicity, cells were treated with void dendrosomes. These experiments showed that bare dendrosomes Den450 and Den700 when exposed to A7r5 cells (rat aortic somatic muscle cells) not only showed no deleterious effects, but even seemed to help their propagation. This surprising effect probably implies the fact that these agents can act as adjuvants and improve uptake of nutrients by cells. Southern blot analysis also clearly demonstrated the episomal presence of the carried gene in the cytoplasm of transfected cells and therefore the capability of dendrosmes in delivery of genes into cells (Sarbolouki et al., 2000). Several advantages of dendrosomes confer them other potentials for use in DNA vaccination. These include protection of plasmid DNA from nuclease degradation, efficient delivery of their contents to antigen presenting cells (APCs) and extended release of cargo. 
Mixing the HBsAg gene-harboring plasmid with a small amount of dendrosomes followed by intramuscular or intradermal administration of the mixture into BALB/C mice, resulted in a much higher production of anti-HBs antibodies compared to the administration of recombinant antigen itself (Sarbolouki et al., 2000). More recent studies by another group on the protective efficiency of dendrosomes as novel nano-sized adjuvants, have also approved their capability for DNA vaccination against allergy (Dutta et al., 2006). Conventional immunotherapies suffer from the drawbacks of use of an active antigen, such as sever IgE-mediated side effects like anaphylactic reactions induced by cross-linking of pre-existing IgE antibodies on the surface of mast cells (Buttgereit et al., 2001). However, use of Den123 for delivery of allergen-encoding plasmid for DNA vaccination, yielded promising results as these nanoparticles showed IgE inhibition while maintaining Th1/Th2 balance after DNA vaccination, sustained release of DNA plasmids and augmentation of the IgG2a level gradually by prolongation of the intracellular presence of the plasmid (Dutta et al., 2006; Balenga et al., 2006). In another study, the dendrosome Den123 was used to deliver and enhance transfection of DNA vaccine plasmid encoding gB gene of Herpes Simplex Virus type- 1 along with Bax-encoding plasmid in order to evaluate the apoptosis induction effect on DNA vaccination efficiency (Pourasgari et al., 2007). Another group has recently synthesized and used dendrosomes containing entrapped PPI dendrimer-DNA complexes in genetic immunization against hepatitis B as well. The dendrosome formulation chosen for this study was DF3 as it possessed optimum size and entrapment efficiency. Animals immunized with PPI dendrimer-DNA complex entrapped within DF3 dendrosomes underwent maximum immune response in terms of total IgG compared to those immunized with plasmid DNA alone and/or PPI dendrimer-DNA complex. Higher level of IFN- $\gamma$ in DF3-immunized animals also suggested that the immune response was strictly Th1-mediated (Dutta et al., 2006). These results are in accordance with our observations about the superiority of dendrosomes in genetic immunization and DNA vaccination compared to other strategies. The dendrosomes DF3 prepared by the reverse phase evaporation method have also been used in transfection of HEK-293 cells with PGL2 showing that they possess a superior transfection against other non-viral delivery systems under study .

In a comparative study, apoptosis induction in human lymphoma and leukemia cell lines was assessed using dendrosomes carrying wild type p53(Dend + p53) along with other very commonly used non-viral carrier lipofectin (Lipo+p53). The rate of apoptosis in Dend+p53 transfected K562 cells (human erythroleukemia cell line) which do not produce the p53 protein (Buttgereit et al., 2001) was twice that of the Lipo+p53 transfected cells, which was indicative of higher transfection efficiency of dendrosomes. In toxicity assessment studies, lipofectin showed a higher cytotoxicity on CCRF and MOIT-4 cells (belonging to T-lymphocyte cell lines) compared to the dendrosomes used (Massumi et al., 2005). Another study reported successful and efficient transfection of A549 (a human lung cell line) by dendrosomes containing the recombinant rotavirus VP2 gene equal to that of lipofectin results where dendrosomes showed a lower cytotoxicity (Pourasgari et al., 2007). In our recently published work, dendrosomes prepared at the IBB center, Iran, were studied and assessed in several aspects including interaction of dendrosomes with plasmid and genomic DNA, their ability in delivery and expression of genes into Huh7, VERO, Bowes, Raw, U-937, CCRF-CEM, MOLT-4 and K562 cells, comparison of their performance with a commercial gene porter lipofectin and bacterial ghosts, their non-toxicity against human cells and animal models and their performance as adjuvant in immunization of BALB/c mice against hepatitis C (Sadeghizadeh et al., 2008). 
CD spectra of the studied dendrosomes entrapping DNA molecules indicated that the commonly used Den123 (made of amphipatic monomers) would moderately cause a transition from B- to A-form in linear DNA. Also, sensitivity of the interaction with the GCcontent of DNA was assessed according to the CD spectra information. Results showed that dendrosomes generally and mildly transform high and low GC-content DNA from B-form into another B-form DNA. Transfection and expression studies also demonstrated that all the dendrosomes used could perform best at very low levels and at weight ratios of Den/DNA ranging from $1 / 1$ to $1 / 10$ therefore minimizing the chance of undesired side effects on the host (Holter \& Fordis, 1989). Superiority of Den55 and Den10 compared to lipofectin and bacterial ghosts was also seen in these studies. Results from non-toxicity and immunization experiments also demonstrated that cells exposed to dendrosomes did not show any signs of toxicity whereas those exposed to lipofectin revealed sever toxicity and that animal immunization with dendrosomes caused long term immunization of mice treated with Den123 carrying the HBsAg or the HCV core pcDNA3 without developing signs of toxicity or discomfort (Sadeghizadeh et al., 2008).

Recently, based on the mentioned desired properties of dendrosomes, our group has employed this system for the delivery of a hydrophobic anticancer agent, curcumin into tumor cell lines (manuscript in preparation). This new formulation of curcumin, hereafter referred to as dendrosomal curcumin, is prepared in a very simple mixing-sonication step, and has been shown to significantly improve curcumin water solubility, an important limiting factor for the use of free curcumin as a chemotherapeutic. Using the intrinsic fluorescence property of curcumin (Bisht et al., 2007) and by fluorescence microscopy, cellular uptake levels of dendrosomal curcumin were shown to increase significantly compared to that of free curcumin, More importantly, using FACS analysis and MTT assays, it was demonstrated that as a result of the treatment of human gastric adenocarcinoma cell line, with dendrosomal curcumin, free curcumin or void dendrosomes in vitro, the rate of apoptosis and cell cycle arrest induced by dendrsomal curcumin was significantly higher than that of free curcumin and that treatment of void dendrosomes induced no sign of toxicity on the cells. Similarly, administration of dendrosomal curcumin into tumor-bearing mice in vivo, abolished tumor progression and toxicological analysis indicated that this novel formulation of curcumin did not cause any severe side effects or cytotoxicity in mice. Following in vitro assays, our group performed experiments to confirm apoptosis induction and tissue uptake of dendrosomal curcumin in vivo. To this end, we injected cancerous cell line WEHI-146 (fibrosarcoma) intraperitoneally into BALB-c mice which led to generation of tumors in mice. Administration of dendrosomal curcumin into the mice gave rise to decrease of size or elimination of tumors. The results of FACS analysis, performed to determine the type of cell death, exhibited the occurrence of apoptosis. In comparison with negative control samples (void dendrosome and curcumin), the results of real-time PCR on genes underlying apoptosis (both apoptosis stimulatory and inhibitory genes) confirmed the induction of apoptosis (manuscript in preparation).

All together, our data suggest that dendrosomes are not only promising gene carriers, but also could be used as efficient drug delivery vehicles for hydrophobic agents.

\subsection{Bacteriophages}

Bactreiophages, also abbreviated as phages, are amongst non-viral biological agents employed for gene delivery. Bacteriophages are the most abundant life forms in the biosphere and exist in various environments as part of a complex microbial ecosystem 
(Clokie et al., 2011). These particles are consisting of a DNA or RNA genome contained within a protein coat. They infect bacteria and either incorporate viral DNA into the host genome, replicating as part of the host (lysogenic cycle), or multiply inside the host cell before releasing phage particles by budding from the membrane or actively lysing the cell (lytic cycle).

They could be found almost in all environments even in the human or animal body. The normal host of routinely used phages as gene delivery vectors (M13 and lambda) is the well characterized bacterium E. coli which is a vital component of the intestinal flora. Therefore it could be suggested that at least some bacteriophage species are stable in the gastro-intestinal tract and should be transferred intact via lymph into blood circulation. If so, this would raise the possibility that phages containing therapeutic genes could be administered orally.

Although still in the early research stages, bacteriophage vectors offer an attractive alternative to various viral and non-viral vectors, because they can potentially overcome the drawbacks of either approach (Table 1). For example, a major advantage of bacteriophages over animal viral vectors is their lack of natural tropism for animal cells, a major concern for current animal viral-vector targeting. Even if non-specific internalization of phage were to occur, the production of phage proteins or replicative phages is unlikely in the foreign milieu of an animal cell (Larocca \& Baird, 2001).

\begin{tabular}{|l|c|c|c|}
\hline & Animal Virus & Synthetic Vector & Phage \\
\hline Potential toxicity & High & Low & Low \\
\hline Generation of competent virus & Yes & No & No \\
\hline Viral protein expressed & Yes & No & No \\
\hline Complexity & High & Low & Low \\
\hline Cost & High & Low & Low \\
\hline Gene transfer efficiency & High & Low & Low \\
\hline Reproducibility & High & Low & High \\
\hline Genetic targeting & Limited & Limited & Yes \\
\hline Directed evolution & No & Yes & Yes \\
\hline
\end{tabular}

Table 1. A comparison of features of phage vectors with animal and synthetic vectors (Larocca \& Baird, 2001).

Also they possess some other advantages. These include the presence of a capsidic structure surrounding the DNA. This is absent in non-viral systems, whose DNA is therefore sensitive to degradation, which impairs gene transfer efficacy, especially in gene therapy experiments performed in vivo (Schmidt-Wolf \& Schmidt-Wolf, 2003 ). The genetic or structural modification of capsid proteins can be easily achieved in phages, allowing selective targeting of eukaroytic receptors and consequent tissue-specific transduction. Furthermore, bacteriophage DNA does not contain eukaryotic sequences and it can be completely replaced by exogenous sequences. These characteristics avoid recombination phenomena, which have contributed to the doubts raised regarding the clinical safety of eukaryotic viruses-derived vectors (Thomas et al., 2003). Phage vector production is expected to be simpler and more cost effective than many existing vectors, because phage can be produced to high titer in the supernatant of bacterial cultures and easily purified at a large scale. The 
baceteriophage has generous packaging capacity beyond what most viral vectors like adenoviruses $(8 \mathrm{~kb})$, adeno-associated viruses $(4.7 \mathrm{~kb})$, or lentiviruses $(8-10 \mathrm{~kb})$ offer (Chauthaiwale et al., 1992). In addition, phages have been experimentally administered to animals and safely used in humans for applications that include the treatment of bacterial infections (Stone, 2002).

The greatest limitation of phage-mediated gene delivery which has restricted its use is low efficiency compared with typical viral vectors. To partially address this problem, one great technological achievement called phage display is utilized. Phage display is based on expressing recombinant proteins or peptides fused to phage coat proteins. The genetic information encoding for the displayed molecule is physically linked to its product via the displaying particle.

Since a major issue in gene therapy is the development of effective vehicles to deliver a therapeutic gene to specific target cells to achieve adequate and sustained selective expression in the diseased tissue, with minimal toxicity in other tissues, many attempts have been prompted in which, by using phage display, natural ligands for cell-surface receptors have been linked to phages to enhance the efficiency and selectivity of gene transduction. In 1999, Larocca et al first described the transduction of a mammalian cell by a genetically targeted filamentous bacteriophage. They modified the phage genome to display fibroblast growth factor on its surface coat as an N-terminal fusion to the minor coat protein pIII, and showed that such phages undergo receptor-mediated endocytosis, resulting in the expression of phage-encoded genes in mammalian cells.

One of appealing characteristics of phage display is to identify novel cell-targeting ligands, which increase the rate and specificity for the transport of macromolecules. Phage-display technology offers large collections of potential ligands including short peptides, antibody fragments and randomly modified physiological ligands able to bind to cell receptors. Thus, novel ligands can be selected from phage libraries by their ability to deliver a reporter gene to targeted cells. With the incorporation of targeting ligands, specificity, delivery and efficiency of cell transduction can be dramatically improved. For example, the specificity of EGF (epidermal growth factor) -targeted phages is expected to be limited to cells expressing EGF receptor, which is elevated in many tumors.

Previous studies have demonstrated targeted gene-delivery to mammalian cells using modified phage-display vectors. Specificity is determined by the choice of the genetically displayed targeting ligand. Without targeting, phage particles have virtually no tropism for mammalian cells. This raises the possibility of developing phage vectors for targeted gene therapy.

\section{Concluding remarks and future perspectives}

It is well established that the key to gene therapy research is development of proper gene carriers. Calcium-phosphate precipitation, use of DEAE, PEIs, PLLs, polybrene/dimethyl sulfoxide, liposomes, cationic amino polymers and dendrimers are major chemical methods used for transfection of genes. However, only liposomes and dendrimers are widely used for efficient in vivo gene delivery and the other methods usually have a lower efficiency and can be applied only in vitro. Lipsomes and dendrimers also have limitations such as fast clearance of liposomes from blood, degradation of their content by lysosomic enzymes as they are phagocytosized, hemolytic toxicity and cytotoxicity of dendrimers owing to their cationic nature and premature release of the dendrimer's cargo in plasma. 
Dendrosomes, however, might be an exception in this regard. Dendrosomes are non-viral nanovehicles that form stable, biodegradable, neutral, readily synthesized hyperbranched spherical nanostructurs. They not only can deliver genes of various sizes and drugs into target cells but also have been proven to be efficient porters for DNA vaccination in vivo. A unique feature of dendrosomes is the ease with which they provide suitable inert gene porters for various DNA sizes and target cells. Dendrosomes may trap DNA molecules within their arms or/and form clusters entrapping DNA inside with no strong electrostatic bonds, but rather with weak interactions with DNA. This is important as most DNA carriers make bonds with DNA and condense it to a proper size for carriage and this reduces the expression of the condensed DNA even when successfully transfected into cells. But, dendrosomes do not condense DNA while entrapping it efficiently enough for transfection both in vitro and in vivo with no toxicity on target cells. However, optimizing the DNA/dendrosome ratio and safe amounts of the carrier is an essential step to be considered.

Bacteriopahges present tantalizing opportunities for therapeutic applications given further research and development. Exploiting the natural properties of these biological entities for specific gene delivery applications will also expand the repertoire of gene therapy vectors available for clinical uses. The discovery that targeted displayed phage can deliver genes to cells has created new opportunities for expanding the use of phage-display in gene discovery and now gene therapy.

\section{Acknowledgements}

This work is dedicated to the lovable and unforgettable memory of the late Dr. Mohammad Nabi Sarbolouki. The authors are deeply grateful to Research Council of Tarbiat Modares University and Center of Excellence of Biotechnology for their help. Also, we kindly acknowledge Dr. Farhood Najafi for his gift giving various kinds of dendrosomes. The current address of A.P is Laboratory of Mitotic Mechanisms and Chromosome Dynamics, Institute for Research in Immunology and Cancer (IRIC), University of Montreal, H3T 1J4, Quebec, Canada.

\section{References}

Alivisatos A (1996). Perspectives on the physical chemistry of semiconductor nanocrystals. J Phys Chem. 100:13226-39.

Balenga NAB, et al (2006). Protective efficiency of dendrosomes as novel nano-sized adjuvants for DNA vaccination against birch pollen allergy. J Biotechnol. 124: 602614 .

Bielinska AV, et al (1997). Regulation of invitro gene expression using antisense oligonucleotides for antisense expression plasmids transfected using starburst PAMAM dendrimers. Nucleic Acid Res. 24: 2176-2184.

Bisht S, Feldmann G, Soni S, Ravi R, Karikar C, Maitra A, Maitra (2007). A polymeric nanoparticle-encapsulated curcumin ("nanocurcumin"): a novel strategy for human cancer therapy. J Nanobiotechnol. Apr 17,5:3.

Bousif $\mathrm{O}$, et al (1995). A versatile vector for gene and oligonucleotide transfer into cells in culture and in vivo. Proc Natl Acad Sci USA. 92: 7294-7301. 
Brannon-Peppas L, Balanchette JO (2004). Nanoparticles and targeted systems for cancer therapy. Adv Drug Deliv Rev. 56: 1649-1659.

Buttgereit P, Weineck S, Ropke G, Marten A, Brand K (2000). Efficient gene transfer into lymphoma cells using adenoviral vectors combined with lipofectin. Cancer Gene Ther. 7:1145-1155.

Buttgereit P, et al (2001). Effects of adeniviral wild-type p53 gene transfer in p53 mutated lymphoma cells. Cancer Gene Ther.8:430-439.

Chauthaiwale VM, Therwath A, Deshpande VV (1992). Bacteriophage lambda as a cloning vector. Microbiol Rev. 56: 577-591.

Clokie M, Millard A, Letarov A, Heaphy S (2011). Phages in nature. Bacteriophage. 1: 31-45.

Dobrovolskaia MA, McNeil AE (2007). Immunological properties of engineered nanomaterials. Nat Nanotechnol. 2: 469-478.

Dutta T, et al (2006). Dendrosome-based gene delivery. J Experimental Nanosci. 1: 235

Felgner PL (1991). Cationic liposome-mediated transfection with lipofectin reagent. Inc: Methods in molecular biology, Vol 7: Gene transfer and expression, edited by Murray EJ. Humana Press Inc. Clifton, New Jersey, United Stated.

Gonzalez H, Hwang SJ, Davis ME (1999). New class of polymers for the delivery of macromolecular therapeutics, Bioconjug Chem. 10:1068- 1074.

Gulick T (2003). Curr Protoc Cell Biol. Transfection using DEAE-dextran. Aug;Chapter 20:Unit 20.4.

Guyden J (1993). Techniques for gene cloning and expression. In: Recombinant DNA technology edited by Steinberg M, PTR Printic Hall, New Jersey, United States, pp: 81-97.

Hammarskjold ML (1991). Manipulation of SV40 vectors. In: Methods in molecular biology, Vol7: Gene transfer and expression protocols edited by Murray EJ Clifton, Humana Press Inc, pp: 169-173.

Hashida M, Takemura S, Nishikawa M, Takakura Y (1998). Targeted delivery of plasmid DNA complexed with galactosylated poly(l-lysine). J Control Release 53:301- 310.

Holter W, Fordis CM, et al (1989). Efficient gene transfer by sequential treatment of mammalian cell with DEAE-Dextran and deoxyribonucleic acid. Exp Cell Res. 184:546-551.

Kawasaki ES, Player A (2005). Nanotechnology, nanomedicine and the development of new therapies for cancer. Nanomedicine. 1: 101-109.

Kichler A (2004). Gene transfer with modified polyethylenimines. J Gene Med. Feb;6 Suppl 1:S3-10.

Kukowska J, et al (1996). Efficient transfer of genetic material into mammalian cells using starburst polyamidoamine dendrimers. Proc Natl Acad Scie USA. 93:4897-4902.

Larocca D, Baird A (2001). Receptor-mediated gene transfer by phage-display vectors: applications in functional genomics and gene therapy. Drug Discov Today. 6: 793801.

Lewin B, Genes IX (2007). Johnes \& Bartlett Publishers, Inc, Oxford University Press, United States, New York.

Li S,Thacker LN (1997). High efficiency stable DNA transfection using cationic detergent and glycerol. Biohem Biophys Res Commun. 231: 531-534. 
Liu G, Molas M, Grossmann GA, Pasumarthy M, Perales JC, Cooper MJ,.Hanson RW (2001). Biological properties of poly-l-lysine-DNA complexes generated by cooperative binding of the polycation, J Biol Chem. 276: 34379-34387.

Massumi M, et al (2005). Apoptosis induction in human lymphoma and leukemia cell lines by transfection via dendrosomes carrying wild type p53 cDNA. Biotechnol Lett. 28: 61-66.

Moghimi SM, Szebeni (2003). Stealth liposomes and long circulating nanoparticles: critical issues in pharmacokinetics, opsonization and protein-binding properties. Prog Lipid Res. 42:463-478.

Nishikawa M, Takemura S, Takakura Y, Hashida M (1998) . Targeted delivery of plasmid DNA to hepatocytes in vivo: optimization of the pharmacokinetics of plasmid DNA/galactosylated poly(l-lysine) complexes by controlling their physicochemical properties. J Pharmacol Exp Ther. 287:408- 415.

Okayama, et al (1991). Calcium phosphate mediated gene transfer into established cell lines. Inc: Methods in molecular biology, Vol 7: Gene transfer and expression, edited by Murray EJ. Humana Press. New Jersey, Clifton, United States.

Pourasgari F, et al (2007). Low cytotoxicity effect of dendrosome as an efficient carrier for rotavirus VP2 gene transferring into a human lung cell line: Dendrosome, as a novel intranasally gene porter. Mol Biol Rep. 2007 Oct 7. [Epub ahead of print].

Robson T, Hirst DG (2003). Transcriptional targeting in cancer gene therapy. J Biomed Biotechnol. 2:110-137.

Roy I, Mitra S, Maitra A, Mozumdar S ( 2003). Calcium phosphate nanoparticles as novel non-viral vectors for targeted gene delivery. Int J Pharm. Jan 2:25-33.

Sadeghizadeh M, et al (2008). Dendrosomes as novel gene porters-III. J Chem Technol Biotechnol. 83:912-920.

Sarbolouki MN, Sadeghizadeh M, Yaghoubi MM, Karami A, Lohrasbi T (2000). Dendrosomes: a novel family of vehicles for transfection and therapy. J Chem Technol Biotechnol. 75:919-922.

Saunak S, et al (2004). Polyvalent dendrimer glucosamine conjugated prevent scar tissue formation. Nat Biotechnol. 22: 977-984.

Schmidt-Wolf G, Schmidt-Wolf I (2003). Non-viral and hybrid vectors in human gene therapy: an update. Trends Mol Med. 9:67-72.

Seow Y, J.Wood M (2009). Biological gene delivery vehicles: beyond viral vectors. Mol Ther. 17: 767-777.

Singelton P, Sainsbury D (1995). Dictionary of microbiology and molecular biology, John Wiley \& Sons, pp: 902-903, UK.

Sinha R, et al (2006). Nanotechnology in cancer therapeutics: bioconjugated nanoparticles for drug delivery. Mol Cancer Ther. 5: 1909-1917.

Stone R (2002). Bacteriophage therapy. Stalin's forgotten cure. Science. 298: 728-731.

Strauss PL, et al (1994). Transfection of mammalian cells via lipofectin. Inc: Methods in molecular biology, Vol 54: YAC protocols, edited by Markie D Totowa. Humana Press Inc.

Suh W, Chung JK, Park SH, Kim SW (2001). Anti-JL1 antibody-conjugated poly(l-lysine) for targeted gene delivery to leukemia T cells. J Control Release 72: 171- 178.

Suntherland A (2002). Qunatum dots as luminescent probes in biological systems. Curr Opin Solid State Mater Sci. 6:360-370. 
Tae B, Park G, Jeong JH, Kim SW (2006). Current status of polymeric gene delivery systems. Advanced Drug Delivery Reviews. 58:467- 486.

Thomas C, Ehrhardt A, Kay M (2003). Progress and problems with the use of viral vectors for gene therapy. Nat Rev Genet. 4:346-358.

Tomalia DA (2005). Birth of a new macromolecular architecture: dendrimers as quantized blocks for nanoscale synthetic polymer chemistry. Prog Polym Sci. 30: 294-324.

Wong TK, Neuman E (1982). Electric field mediated gene transfer. Biochem Biophys Res Commun. 107: 584-587.

Zhang L, Granick S (2006). How to stabilize phospholipids liposome (using nanoparticle) Nano Lett. 6:694-698.

Zhang L, et al (2007). Nanoparticles in medicine: therapeutic applications and developments. Clinic Pharmacol Therapeutics. Doi:10.1038/sj.clpt.6100400. 


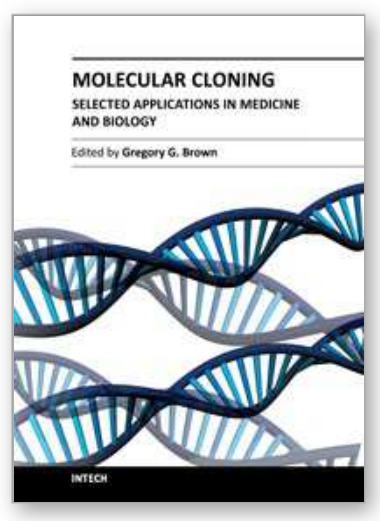

\author{
Molecular Cloning - Selected Applications in Medicine and Biology \\ Edited by Prof. Gregory Brown
}

ISBN 978-953-307-398-9

Hard cover, 324 pages

Publisher InTech

Published online 12, October, 2011

Published in print edition October, 2011

The development of molecular cloning technology in the early 1970 s created a revolution in the biological and biomedical sciences that extends to this day. The contributions in this book provide the reader with a perspective on how pervasive the applications of molecular cloning have become. The contributions are organized in sections based on application, and range from cancer biology and immunology to plant and evolutionary biology. The chapters also cover a wide range of technical approaches, such as positional cloning and cutting edge tools for recombinant protein expression. This book should appeal to many researchers, who should find its information useful for advancing their fields.

\title{
How to reference
}

In order to correctly reference this scholarly work, feel free to copy and paste the following:

Abbas Padeganeh, Mohammad Khalaj-Kondori, Babak Bakhshinejad and Majid Sadeghizadeh (2011). NonViral Vehicles: Principles, Applications, and Challenges in Gene Delivery, Molecular Cloning - Selected Applications in Medicine and Biology, Prof. Gregory Brown (Ed.), ISBN: 978-953-307-398-9, InTech, Available from: http://www.intechopen.com/books/molecular-cloning-selected-applications-in-medicine-and-biology/nonviral-vehicles-principles-applications-and-challenges-in-gene-delivery

\section{INTECH}

open science | open minds

\section{InTech Europe}

University Campus STeP Ri

Slavka Krautzeka 83/A

51000 Rijeka, Croatia

Phone: +385 (51) 770447

Fax: +385 (51) 686166

www.intechopen.com

\section{InTech China}

Unit 405, Office Block, Hotel Equatorial Shanghai

No.65, Yan An Road (West), Shanghai, 200040, China

中国上海市延安西路65号上海国际贵都大饭店办公楼405单元

Phone: +86-21-62489820

Fax: $+86-21-62489821$ 
(C) 2011 The Author(s). Licensee IntechOpen. This is an open access article distributed under the terms of the Creative Commons Attribution 3.0 License, which permits unrestricted use, distribution, and reproduction in any medium, provided the original work is properly cited. 